\title{
Lepton Flavour Violation Experiments
}

\author{
Ajit Kurup*† \\ Imperial College London \\ E-mail: a.kurup@imperial.ac.uk
}

This paper gives an overview of current and future charged lepton flavour violation experiments that are muon based.

16th International Workshop on Neutrino Factories and Future Neutrino Beam Facilities 25 -30 August, 2014

University of Glasgow, United Kingdom

\footnotetext{
* Speaker.

${ }^{\dagger}$ The author would like to thank the AlCap, COMET, DeeMe, MEG, Mu2e, Mu3e and PRISM collaborations for providing the material presented here.
} 


\section{Introduction}

The observation of neutrino oscillations shows that lepton flavour number is not conserved in the neutral lepton sector of the Standard Model (SM). This leads us to consider lepton flavour number violation in the charged lepton sector, for example, modes such as: $\mu^{+} \rightarrow e^{+}+\gamma ; \mu^{-}+$ $N(A, Z) \rightarrow e^{-}+N(A, Z) ; \mu^{+} \rightarrow e^{-}+e^{+}+e^{+} ; \tau \rightarrow \mu \gamma ; \tau \rightarrow 3 \mu$; and $\tau \rightarrow 3 e$.

Incorporating neutrino oscillations into the SM allows charged lepton flavour violation (CLFV) to occur but at rates $<O\left(10^{-52}\right)$ which is about 30 orders of magnitude smaller than what we could expect to measure with a next generation experiment. However, many beyond the SM (BSM) theories predict rates much higher than this $<O\left(10^{-15}\right)$, so measurments of any of the above modes would be a ground-breaking indication of physics beyond the SM.

Advances in accelerator technology have lead to significant improvements in muon beam intensities and quality making muon based searches a very powerful probe of BSM physics. The following sections outline the various muon based experiments exploring this promising area of physics. For further details please see the experiment specific papers in these proceedings.

\section{Muon to Electron Conversion}

Muon to electron conversion can be searched for by looking at muonic atoms where the muon in orbit around the nucleus can convert into an electron via a virtual particle exchange with the nucleus. Intense muons beams $\left(>10^{11}\right.$ muons/s) can be produced as a secondary product of the interaction of an intense proton beam (energy around $8 \mathrm{GeV}$ ) with a target typically made from carbon or tungsten. The proton interaction primarily produces pions which then decay into muons. The beam line also requires a capture and decay magnetic field which provides a channel for the pions to decay to muons whilst keeping the beam focussed. These muons are then stopped in a material, e.g. aluminium, to form muonic atoms. The muons in orbit around the nucleus can then either: decay normally, which is reffered to as decay in orbit (DIO); undergo nuclear capture with subsequent particle emission as the nucleus deexcites; or it can convert into an electron, which is ejected and the nucleus coherently recoils.

\subsection{COherent Muon to Electron Transition Experiment (COMET)}

The COherent Muon to Electron Transition (COMET) Experiment, based at J-PARC in Japan, aims to achieve a single event sensitivity of $2.6 \times 10^{-17}$. COMET will be constructed in two stages, with the ability to search for muon to electron conversion in both stages. COMET Phase-I will allow performance measurements of the beam line, which will be very important to understand sources of backgrounds for COMET Phase-II and it will be used to prototype the detector technology for Phase-II, in addition to searching for $\mu^{-}+N(A, Z) \rightarrow e^{-}+N(A, Z)$ with the cylindrical drift chamber detector. Figure 1 shows the schematic of COMET Phase I and Phase-II. The experiment is composed of a continuous solenoid channel with bending sections to select the charge and momentum of particles. The tracker consists of 5 planes of straw tubes $48 \mathrm{~cm}$ apart with 2 views ( $\mathrm{x}$ and y) per plane and 2 layers per view (staggered by one straw radius). The gas filled straws have a diameter of $9.75 \mathrm{~mm}$ and are made from $12 \mu \mathrm{m}$ thick metalised polyimide with a gold coated tungsten anode wire. The tracker will have a $0.4 \%$ momentum and a $700 \mu \mathrm{m}$ spatial resolution. The 

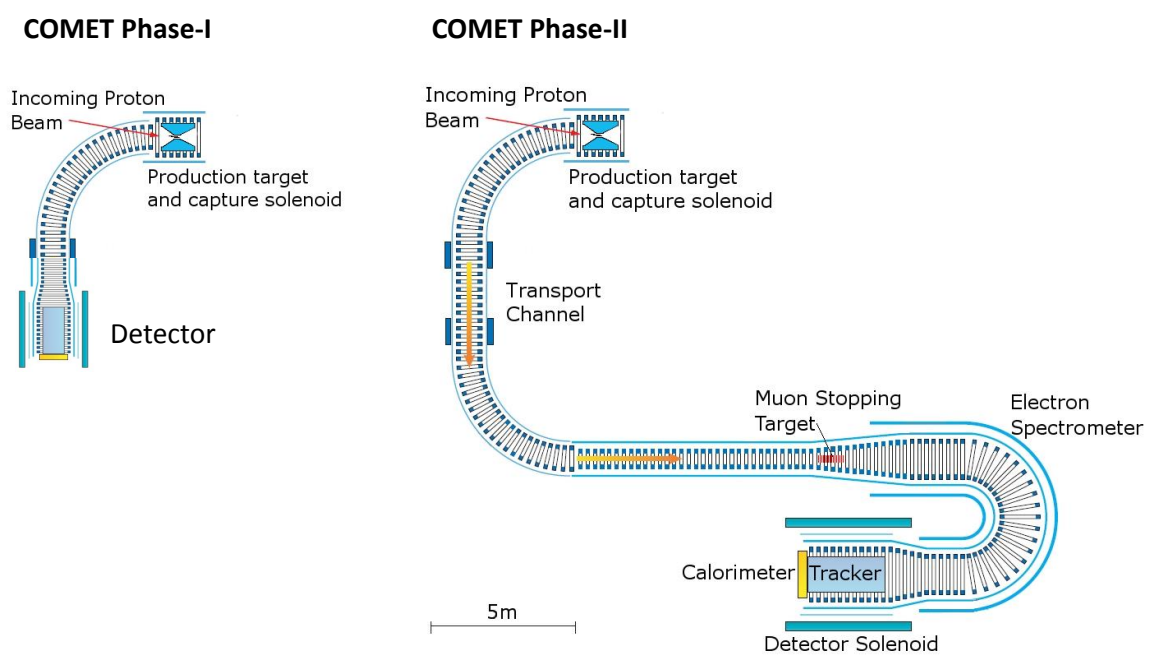

Figure 1: Schematic diagram of COMET Phase-I (left) and Phase-II (right).

calorimeter will be made of an inorganic scintillator and will be used to measure energy, provide particle identification and timing information. Two candidate inorganic scintillator materials were considered: Cerium-doped Lutetium Yttrium Orthosilicate (LYSO); and Cerium-doped Gd2SiO5 (GSO). The readout will be done using APD photo detectors with custom pre-amps. Beam tests have been performed of these crystals to determine their performance and based on this the decision to use LYSO was made.

The electron spectrometer removes unwanted low momentum electrons and lowers the rate in the detectors.

COMET Phase-I is currently under construction and will take data by 2017 and Phase-II will begin construction in 2017 with data taking in 2020 .

\subsection{Mu2e}

The Mu2e experiment, based at Fermilab in USA, aims to achieve a single event sensitivity of $2.4 \times 10^{-17}$. Figure 2 shows a schematic of the experiment which is composed of a continuous solenoid channel starting from $4.7 \mathrm{~T}$ at the production target to $1 \mathrm{~T}$ in the detector region. The detector uses a straw tube tracker to measure the momentum and a scintillating crystal calorimeter for timing and background rejection.

The tracker is composed of metalised mylar tubes $15 \mu \mathrm{m}$ thick with an outer diameter of $5 \mathrm{~mm}$. Two layers of 48 straws are arranged to make a panel; six rotated panels make a plane; two rotated planes make a station; and 20 stations form the tracker.

The calorimeter is made of two annular rings of $\mathrm{BaF}_{2}$ crystals. Each crystal has a hexagonal cross-section, side length is $33 \mathrm{~mm}$, and a length of $180 \mathrm{~mm}$. The readout will be done with either APDs or SiPMs. The goals of the calorimeter is to provide timing of $<1 \mathrm{~ns}$, PID, background rejection, alternate track seed and possible calibration trigger.

Prototyping of the super-conducting solenoids has been performed and procurement of the final solenoids is in progress. The construction of Mu2e is due to commence in 2015 with data 


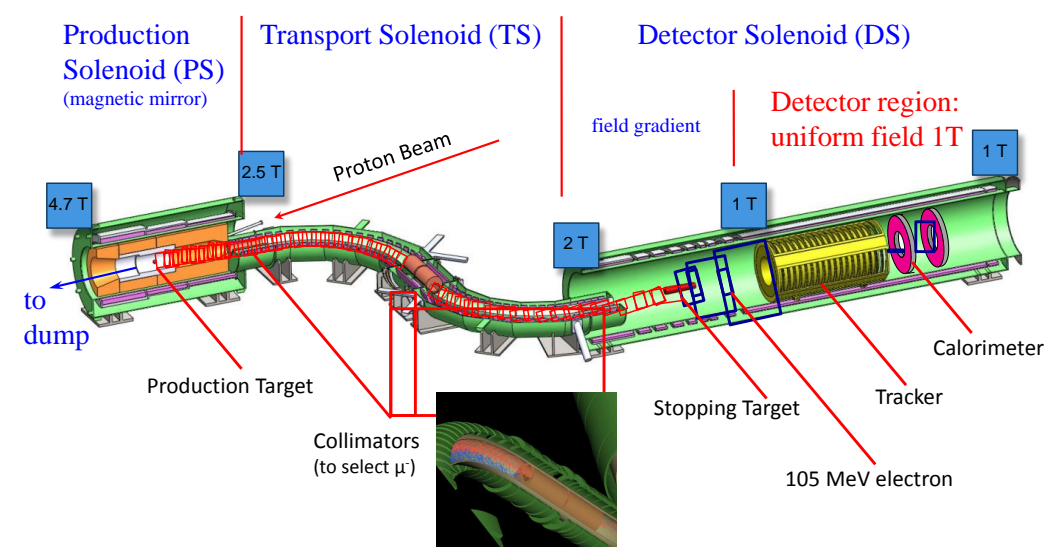

Figure 2: Schematic diagram of Mu2e.

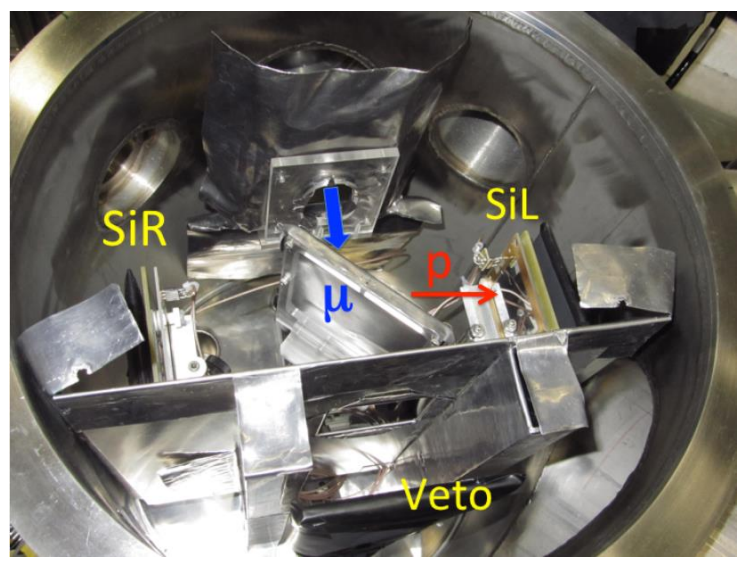

(a)

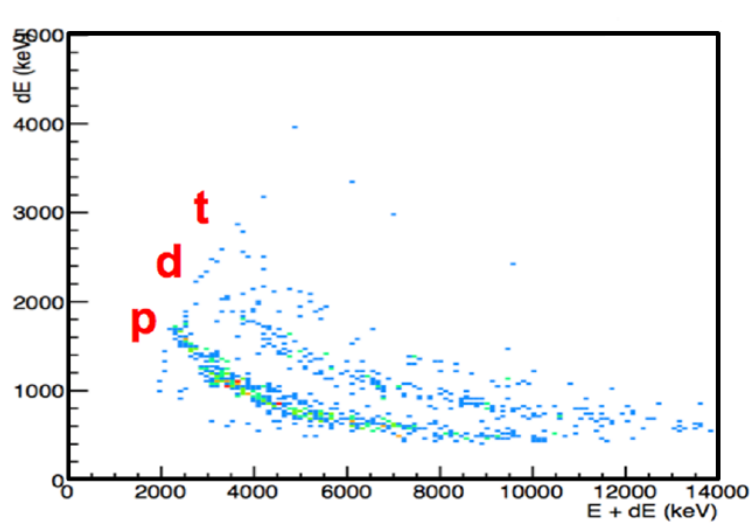

(b)

Figure 3: Picture of the AlCap experiment (a). Initial analysis of results from the data run in December 2013 (b).

taking around 2021.

\subsection{AlCap}

AlCap is a joint experiment at PSI between COMET and Mu2e. The aim is to measure particle emission rates for muon nuclear capture in Aluminium, which are important backgrounds for measuring $\mu^{-}+N(A, Z) \rightarrow e^{-}+N(A, Z)$. Figure $3 \mathrm{a}$ is a picture of the experiment chamber indicating the incoming muon beam, direction of protons emitted by the aluminium target and the silicon detectors and veto. Figure 3b shows the initial analysis of results from the run in December 2013.

\subsection{DeeMe}

DeeMe is an experiment based at J-PARC that aims to measure $\mu^{-}+N(A, Z) \rightarrow e^{-}+N(A, Z)$ with a sensitivity $<10^{-13}$. The experimental concept is different to that of COMET and Mu2e in that: the Silicon Carbide production target used to produce muons is also used to stop the muons; it uses 


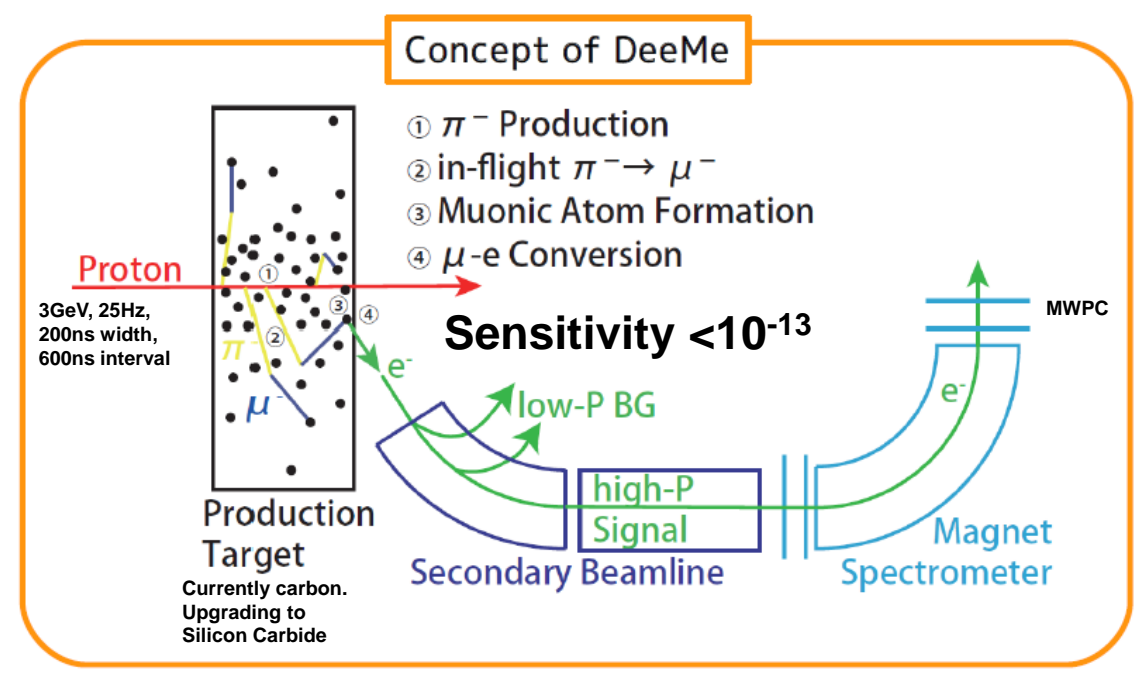

Figure 4: Schematic of the DeeMe experiment.

dipoles for momentum selection; and the proton beam energy is $3 \mathrm{GeV}$. Figure 4 shows a schematic of the experiment. The experiment is under construction, with several magnets of the beam line already delivered.

\subsection{The Phase Rotated Intense Source of Muons (PRISM) Experiment}

The Phase Rotated Intense Source of Muons (PRISM) experiment will be able to achieve a sensitivity of $<10^{-19}$ by rotation of the energy-time phase space, i.e. exchanging the large spread in energy and small spread in time for a large spread in time and small spread in energy. The small energy spread allows a more precise target thickness. There are a few accelerator challenges that need to be solved first before the PRISM beam line, see figure 5, can be realised. These issues are being addressed by the PRISM task force.

\section{Muon to a Positron and a Photon}

The search for $\mu^{+} \rightarrow e^{+}+\gamma$ was the first CLFV mode to be looked for by Hincks and Pontecorvo in 1947 [1]. With modern intense muon beams, significant progress has been made in reducing the limit on the branching fraction of this mode. Positive muon beams are stopped in a thin target and then back-to-back decays are looked for where $\mathrm{E}_{e}=\mathrm{E}_{\gamma}=52.8 \mathrm{MeV}$, since the muon decays at rest. Measurements of this process are dominated by accidental backgrounds, which increases with the muon beam rate. Radiative muon decay also is a background for this but the rate is about $10 \%$ that of accidental backgrounds. Figure 6 illustrates the signal and main background processes.

\subsection{The MEG Experiment}

The MEG experiment, based at PSI, utilises an intense, low momentum $\left(P_{\mu}=28 \mathrm{MeV} / \mathrm{c}\right)$ positive muon beam which is stopped in a thin $\mathrm{CH}_{2}$ target. The rate of stopped muons is $3 \times$ 


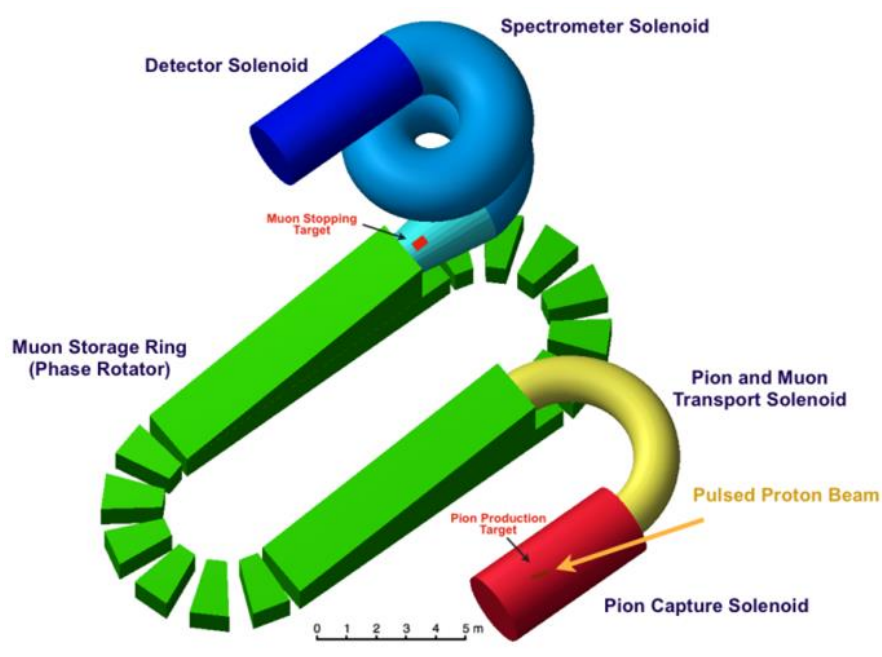

Figure 5: Concept of the PRISM experiment.
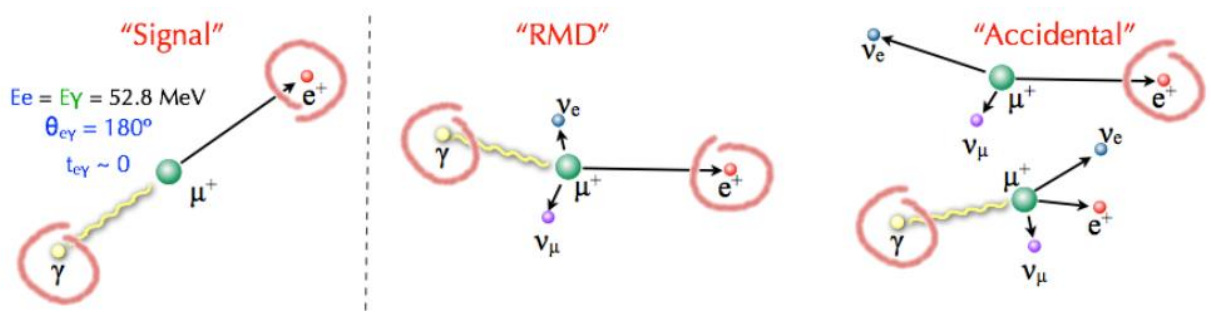

Figure 6: Signal and background processes for $\mu^{+} \rightarrow e^{+}+\gamma$.

$10^{7} \mu^{+} / \mathrm{s}$. The positron emitted from the target is then detected by a low mass drift chamber and the photon is measured by a liquid xenon scintillation calorimeter, see figure 7 .

A blind analysis is performed based on five observables: the energy of the photon $\mathrm{E}_{\gamma}$; the energy of the positron $\mathrm{E}_{e}$; the time between the photon and positron $\mathrm{t}_{e \gamma}$; and the angles between the positron and the photon $\theta_{e \gamma}$ and $\phi_{e \gamma}$. The most recent result, published in 2013 [2], was achieved using data collected from 2009 to 2011 . The upper limit of the branching ratio for $\mu^{+} \rightarrow e^{+}+\gamma$, at the $90 \%$ confidence level, was determined to be $5.7 \times 10^{-13}$, see figure ??.

The MEG experiment will be upgraded to achieve a sensitivity of $5 \times 10^{-14}$ primarily by

- increasing the stopped $\mu^{+}$rate to $7 \times 10^{7} \mu / \mathrm{s}$,

- reducing the target thickness to $140 \mu \mathrm{m}$,

- improving the positron tracking, and 


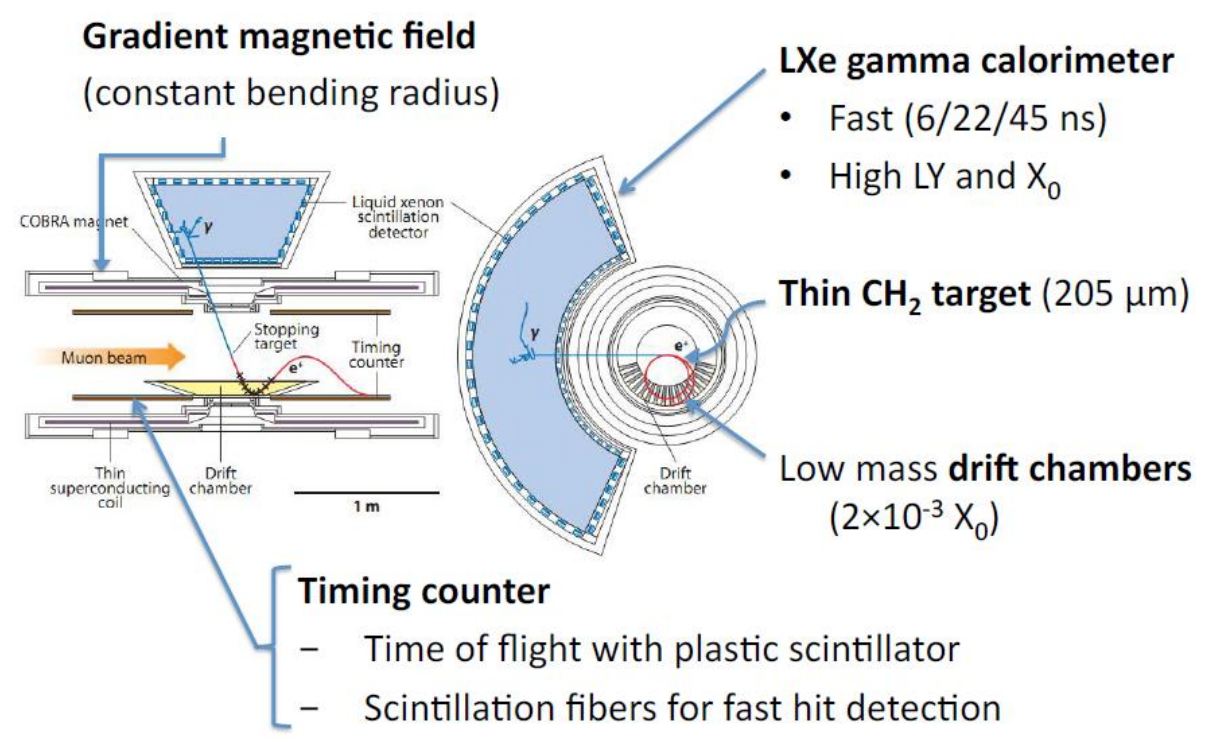

Figure 7: Layout of the MEG experiment.

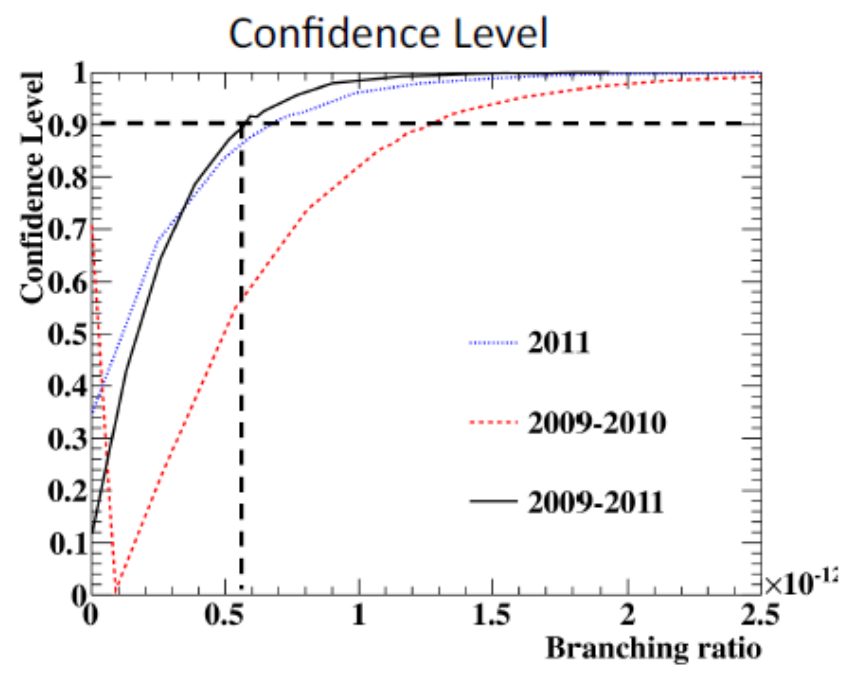

Figure 8: Result of the limit on the branching fraction for $\mu^{+} \rightarrow e^{+}+\gamma$. Publised in 2013 using data taken in 2009 to 2011. 


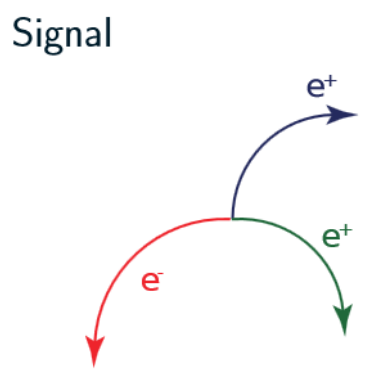

Backgrounds
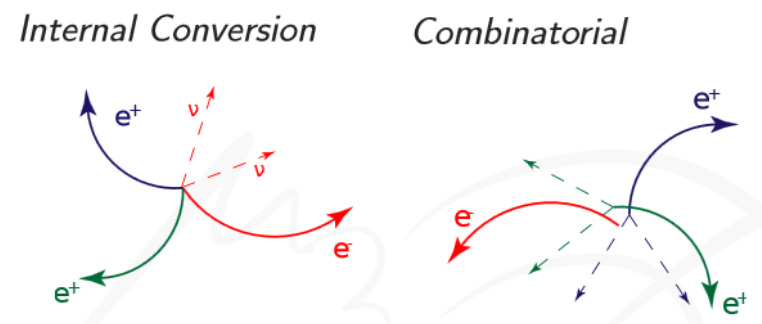

Figure 9: Signal and background processes for $\mu^{+} \rightarrow e^{-}+e^{+}+e^{+}$.

- increasing the coverage and resolution of the calorimeter.

Development of technology for the upgrade is currently ongoing with construction starting in 2015 and data taking starting in 2016.

\section{Muon to an Electron and Two Positrons}

In order to search for $\mu^{+} \rightarrow e^{-}+e^{+}+e^{+}$, positive muons are stopped in a thin target and the three decay products are tracked to a common vertex and the sum of their momenta is required to be zero. The most common backgrounds sources are: internal conversion, which is a normal muon decay with a photon that is converted to an $e^{+} e^{-}$internally; and accidentally combining unrelated events. Figure 9 illustrates the signal and background processes. The sum of the momenta will not be zero and for combinatorial backgrounds and internal conversion. Combinatorial backgrounds will also not have all the decay products produced at the same time. This places strong constraints on the position and timing resolutions of the detector systems. The current World's best limit of the branching ratio for $\mu^{+} \rightarrow e^{-}+e^{+}+e^{+}$is $<1 \times 10^{-12}$ and was set by the Sindrum experiment [3].

\subsection{The Mu3e Experiment}

The Mu3e experiment, based at PSI, aims to measure the branching ratio of $\mu^{+} \rightarrow e^{-}+e^{+}+$ $e^{+}$with a sensitivity of $1 \times 10^{-14}$ to $1 \times 10^{-16}$, for an expected muon decay rate of $>10^{9} \mu^{+} / \mathrm{s}$. A schematic of the experiment is shown in figure 10. The decay products are required to have a momentum of $<53 \mathrm{MeV} / \mathrm{c}$, which places stringent requirements on the amount of material that the tracks pass through, in order to make accurate measurements of momentum and vertexing. The detector systems are: pixel layers; scintillating fibres; and scintillating tiles. The pixel tracker is made from $25 \mu \mathrm{m}$ kapton with $50 \mu \mathrm{m}$ silicon sensors, which is approximately $1 \%$ of a radiation length, see figure 11. The scintillating fibres have 3-5 layers with SiPM and custom ASIC readout, which can achieve a timing resolution of $\sim 1 \mathrm{~ns}$. The scintillating tile tracker is composed of a series of $0.5 \mathrm{~cm}^{3}$ tiles to make a cyclindrical station $12 \mathrm{~cm}$ in diameter and $36 \mathrm{~cm}$ long, see figure 12 . Together with the same readout as the fibres this can achieve a timing resolution of $80 \mathrm{ps}$. 


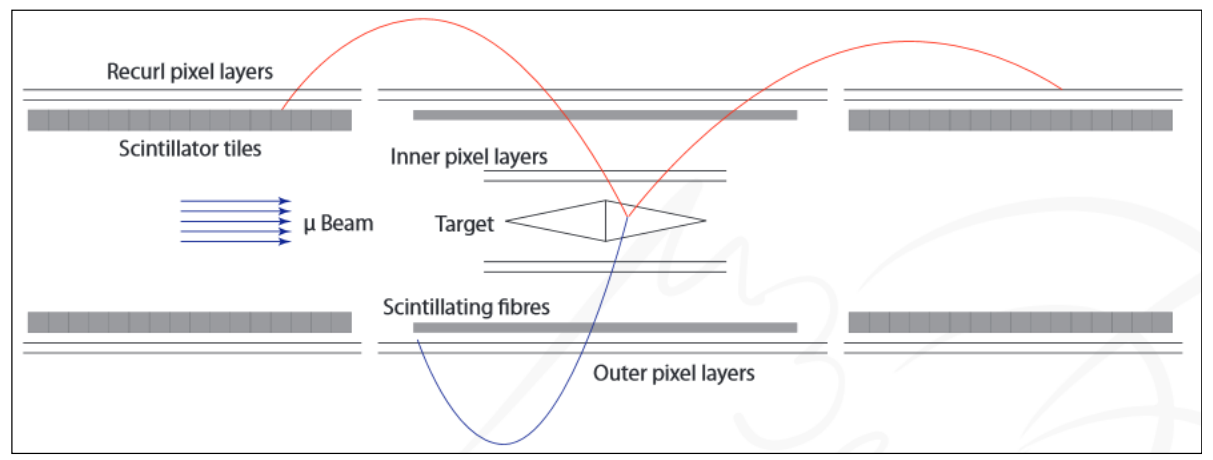

Figure 10: Schematic diagram of the Mu3e experiment.
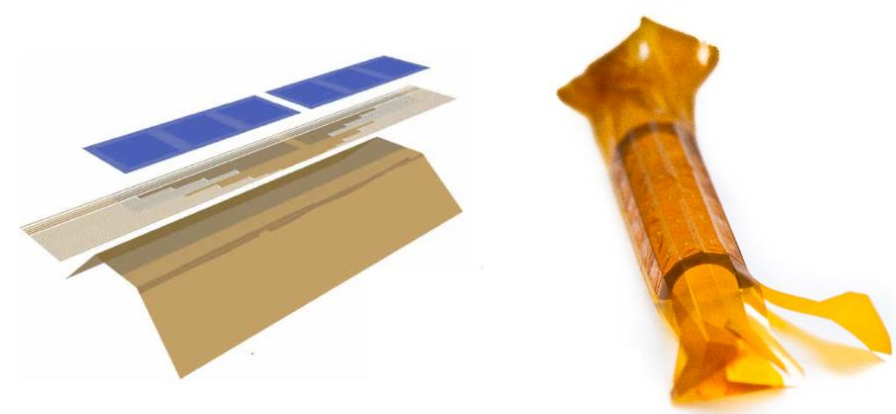

Figure 11: Prototype of the Mu3e pixel tracker.

Tile Station

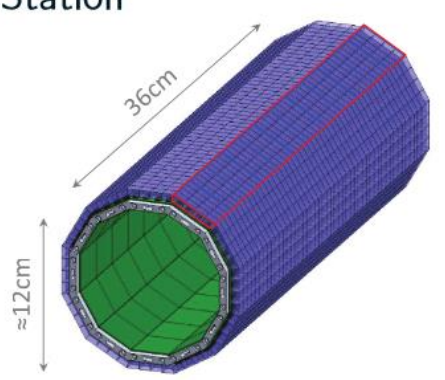

Tile Prototype

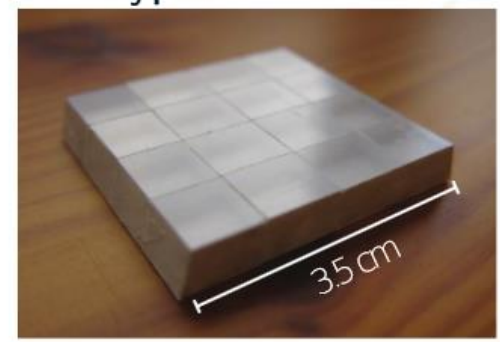

Figure 12: Schematic diagram of a scintillating tile station (left) and a picture of a prototype section (right). 


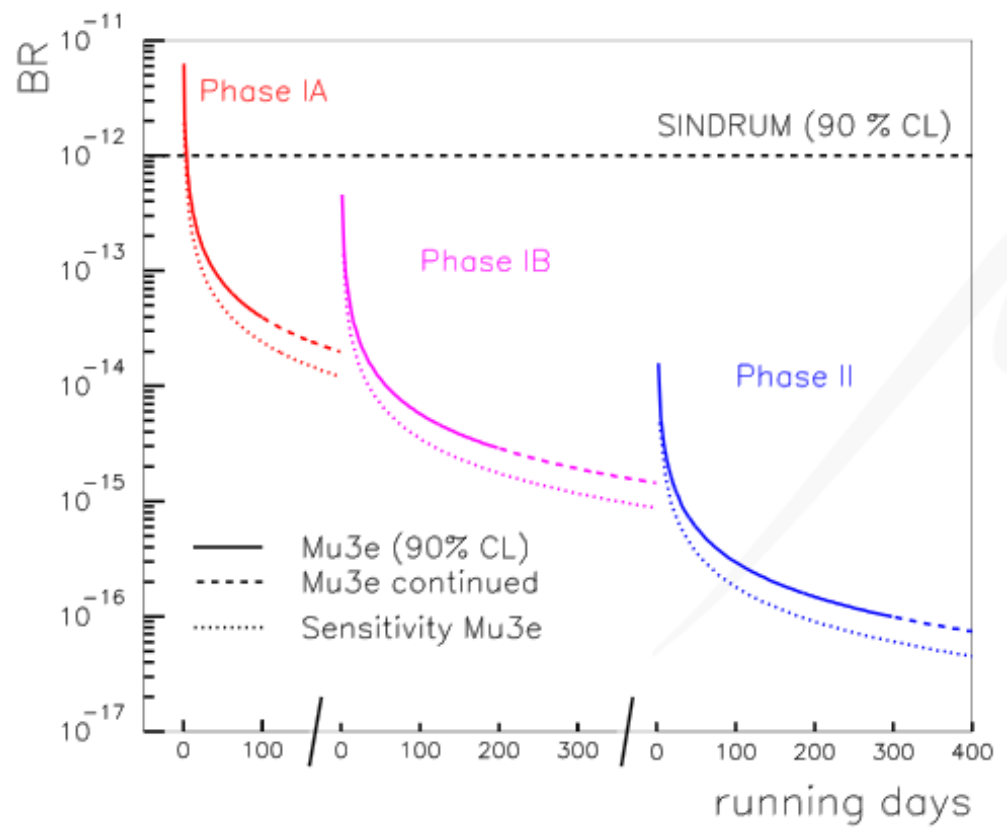

Figure 13: Expected sensitivity for the various phases of Mu3e.

The experiment schedule will be phased, with Phase-IA starting around 2016 with just the central pixel layers and $2 \times 10^{7} \mu / \mathrm{s}$; Phase-IB in 2017 with the first recurl station and $1 \times 10^{8} \mu / \mathrm{s}$; and Phase-II in 2019 with the full detector and $2 \times 10^{9} \mu / \mathrm{s}$. The corresponding estimated sensitivities are shown in figure 13.

\section{Summary}

There are several upcoming experiments that will significantly improve the sensitivity for the CLFV modes $\mu^{+} \rightarrow e^{+}+\gamma, \mu^{-}+N(A, Z) \rightarrow e^{-}+N(A, Z)$ and $\mu^{+} \rightarrow e^{-}+e^{+}+e^{+}$. This will provide a very strong probe of BSM theories and promises a very exciting discovery potential.

Significant progress has been made towards developing the technology for these experiments (including upgrades) and significant results are expected within the next few years.

\section{References}

[1] "Search for Gamma-Radiation in the 2.2-Microsecond Meson Decay Process", E. P. Hincks and B. Pontecorvo, Phys. Rev. 73, 257-258, (1948).

[2] "New Constraint on the Existence of the $\mu^{+} \rightarrow e^{+} \gamma$ Decay", MEG collaboration, Phys. Rev. Lett. 110, 201801, (2013).

[3] "Search for the decay $\mu^{+} \rightarrow e^{+} e^{+} e^{-"}$, SINDRUM Collaboration, Nucl. Phys. B 299(1),1-6, (1988). 